\title{
Tumour necrosis factor: out of my heart!
}

\author{
Lars Vereecke, ${ }^{1,2}$ Dirk Elewaut ${ }^{1,2}$
}

Increasing evidence points to cardiovascular comorbidities in patients with rheumatic diseases, particularly in rheumatoid arthritis (RA) and spondyloarthritis (SpA). ${ }^{1}$ Ischaemic heart disease, valvular involvement including insufficiency and stenosis and arrhythmias can be observed as typical manifestations. They are a major cause of increased morbidity and mortality. While the epidemiological evidence is overwhelming, the underlying mechanisms why chronic inflammatory diseases affect the cardiovascular system are only partially understood. Treatment with anti-tumour necrosis factor (TNF) biologics ameliorates the risk for these comorbidities. ${ }^{1}$ However, insights into the underlying mechanisms and information on responsible cell-type linking arthritis and cardiac disease are yet unclear.

In this issue, Ntari et al describe spontaneous cardiac pathology in a preclinical model of arthritis driven by chronic human TNF expression. They show that TNF signalling in mesenchymal cells is both necessary and sufficient for the development of heart valve pathology in the presented mouse model. ${ }^{2}$

Human TNF transgenic mice which develop spontaneous arthritis (Tg197 line $)^{3}$ were backcrossed to colVI-Cre TNFR $1^{\mathrm{fl} / \mathrm{fl}}$ versus TNFR $1^{\text {cneo/cneo }}$ mutant mice. This approach permits selective mesenchymal-specific genetic ablation versus reactivation of TNF/TNF-receptor 1 (TNFR1), including valve interstitial cells (VICs) of the heart. The same group previously showed that TNF signalling in mesenchymal cells is sufficient to drive chronic gut and joint pathology in an alternative TNF-overexpressing mouse model. ${ }^{4}$ In humans, synovial fibroblasts in the joints and intestinal mesenchymal cells in the gut regulate pathological mechanisms during arthritis ${ }^{5}$ and inflammatory bowel disease (IBD), ${ }^{6}$ which indicates a

\footnotetext{
'Department of Rheumatology, University Hospital Gent, Gent, Belgium

${ }^{2}$ VIB Inflammation Research Center, Unit for Molecular Immunology and Inflammation, Ghent University, Ghent, Belgium
}

Correspondence to Dr Dirk Elewaut, Department of Rheumatology, University Hospital Gent, Gent 9000, Belgium; dirk.elewaut@ugent.be central role for mesenchymal cells in the aetiology of these diseases.

Under chronic TNF exposure, the authors show development of spontaneous left-sided heart valve pathology with fibrosis and thickening of the aortic and mitral valves. This was linked to activated and hyperproliferating VICs. Valvular stenoses develop which leads to degeneration and left ventricular dysfunction. All these features mimic very well the cardiac abnormalities detected in patients with RA and SpA. Using a number of complementary strategies the investigators pinpoint this to a role of TNFR1 on mesenchymal cells. Mechanistically, VICs ex vivo exhibit an activated phenotype, increased TNF production and enhanced proliferative and migratory features. This mirrors abnormalities found in other stromal cell compartments such as synovial fibroblasts in the joint. RNA sequencing of VICs and synovial fibroblasts from huTNF transgenic mice were compared and were shown to display similar dysregulated pathways, including nuclear factor (NF)- $\kappa \mathrm{B}$ signalling and pathways regulating extracellular matrix remodelling and deregulated growth, together indicating an inflammatory and profibrotic phenotype.

One of the intriguing findings of the study is the development of pathological alterations localised in the left side of the heart, particularly the aortic and mitral valvular area while the pulmonary valve as well as blood arteries and vessels appeared unaffected. This is of particular interest given that mesenchymal VICs are equally distributed in both the left and right sides of the heart. The authors suggest that this reflects regions of enhanced biomechanical stress in the heart, in line with the main pump function of left ventricle. This mirrors earlier findings of inflammation at mechanical strain-sensitive entheseal sites in another TNF-driven model of $\mathrm{SpA}$, where biomechanical unloading of the limbs prevents the development of enthesitis. ${ }^{7}$ In line with this, the present study suggests sites of enhanced cardiovascular stress to be particularly sensitive to localised tissue remodelling and development of valvular abnormalities.
However, unlike in joint tissues, inflammation in aortic and mitral valve appeared to be minimal since no massive immune cell recruitment could be observed. This points to differences of inflammatory cells to enter these heart structures under high velocity or could be due to specific structural features of valve which disfavours immune cell accumulation. Alternatively, it could indicate a rapid clearance of inflammatory cells which is less likely. Despite low immune cell numbers, mesenchymal cells are sufficient to drive inflammatory signalling and orchestrate profound structural remodelling, ultimately leading to fibrosis. This is intriguing as tissue fibrosis is not a typical finding in RA and $\mathrm{SpA}$ at synovial sites where accumulation of inflammatory cells and stromal cell hyperplasia seems to dominate along with secondary destruction of articular structures. These findings indicate that synovial fibroblasts and VICs drive TNF-dependent joint and heart pathology, respectively, through site-specific pathological mechanisms. This was also reflected in different expression profiles in VICs versus synovial fibroblasts, which only partially overlapped (40\% of genes), especially genes linked to TNF and NF- $\kappa \mathrm{B}$ signalling were commonly dysregulated, as well as several chemokines.

While this study suggests that mesenchymal expression of TNFR1 is sufficient to drive TNF-mediated heart disease, the study does not rule out contribution of other cell types including endothelial and myeloid cells to heart pathology. In this regard it is interesting to note that recent studies have highlighted a critical role for dendritic cell subsets as well as macrophages $^{8-10}$ in preserving normal cardiac function under ischaemic conditions. As these cells not only produce but also respond to TNF, it is likely that TNFR1 signalling may also be very important on these cell types in the context of TNF-driven cardiovascular disease. ${ }^{11}$

Although the paper convincingly demonstrates the contribution of mesenchymal cells to TNF-driven heart pathology in the presented mouse model, questions remain to what extent arthritis and heart pathology develop independently in response to high hTNF levels, or alternatively, whether these pathologies are mechanistically linked. To address this question, specific synovial fibroblast or VICs TNFR reactivation, instead of general mesenchymal reactivation, in order to evaluate the selective development of arthritis versus cardiac pathology could be very interesting. In this respect, the mechanistic link between 
mesenchymal driven joint and gut pathology in an alternative TNF-driven mouse model is equally intriguing ${ }^{4}$ as gut inflammation is a common comorbidity in SpA. ${ }^{12}$ Interestingly, also IBD is linked to increased cardiac pathology. ${ }^{13}$ Together, these data suggest that intestinal, joint and heart disease can either develop independently in response to chronic inflammation (TNF exposure) or that the aetiology of these diseases is mechanistically closely intertwined.

From a therapeutic perspective, the data illustrate the importance of early detection of cardiovascular morbidity in patients suffering from inflammatory rheumatic diseases and the impact of tight disease control. This underscores the relevance of early intervention and treat-to-target regimens on long-term outcome; not only in joint but also in the cardiovascular system. ${ }^{14}{ }^{15}$ Since human TNF is known to only bind mouse TNFR1, not TNFR2, the data in this paper solidify the crucial role of TNFR1 signalling on the inflammatory features exerted by TNF. In contrast, there is growing evidence that TNFR2 signalling exerts immune regulating properties mainly through its impact on T regulatory cells. ${ }^{16-19}$ Thus the interaction of TNF-TNFR1 appears to be the main driver of proinflammatory pathways during the development of arthritis and cardiac abnormalities. ${ }^{4}$ Therefore, strategies to neutralise the detrimental effects of TNF through TNFR1-selective antibodies may be interesting alternate strategies of TNF blockade. ${ }^{20}$ Overall, the paper by Ntari et al shows a crucial contribution of the mesenchymal cells to the development of heart pathology in a TNF-driven mouse arthritis model with many similarities to human disease.
Handling editor Josef S Smolen

Contributors LV and DE wrote the article jointly.

Funding The authors have not declared a specific grant for this research from any funding agency in the public, commercial or not-for-profit sectors.

Competing interests None declared.

Patient consent Not required.

Provenance and peer review Commissioned; externally peer reviewed.

(C) Article author(s) (or their employer(s) unless otherwise stated in the text of the article) 2018. All rights reserved. No commercial use is permitted unless otherwise expressly granted.

\section{Check for updates}

To cite Vereecke L, Elewaut D. Ann Rheum Dis 2018;77:793-794.

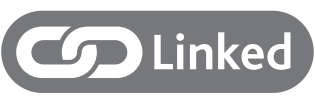

- http://dx.doi.org/10.1136/annrheumdis-2017212597

Ann Rheum Dis 2018:77:793-794.

doi:10.1136/annrheumdis-2018-213118

\section{REFERENCES}

1 Nurmohamed MT, Heslinga M, Kitas GD. Cardiovascular comorbidity in rheumatic diseases. Nat Rev Rheumatol 2015;11:693-704.

2 Ntari L, Sakkou M, Chouvardas P, et al. Comorbid TNFmediated heart valve disease and chronic polyarthritis share common mesenchymal cell-mediated aetiopathogenesis. Ann Rheum Dis 2018.

3 Keffer J, Probert L, Cazlaris H, et al. Transgenic mice expressing human tumour necrosis factor: a predictive genetic model of arthritis. Embo J 1991:10:4025-31.

4 Armaka M, Apostolaki M, Jacques P, et al. Mesenchymal cell targeting by TNF as a common pathogenic principle in chronic inflammatory joint and intestinal diseases. J Exp Med 2008;205:331-7.

5 Ospelt C. Synovial fibroblasts in 2017. RMD Open 2017:3:e000471.

6 Algeri M, Conforti A, Pitisci A, et al. Mesenchymal stromal cells and chronic inflammatory bowel disease. Immunol Lett 2015;168:191-200.
7 Jacques $\mathrm{P}$, Lambrecht S, Verheugen E, et al. Proof of concept: enthesitis and new bone formation in spondyloarthritis are driven by mechanical strain and stromal cells. Ann Rheum Dis 2014;73:437-45.

8 Clemente-Casares X, Hosseinzadeh S, Barbu I, et al. A CD $103{ }^{+}$Conventional Dendritic Cell Surveillance System Prevents Development of Overt Heart Failure during Subclinical Viral Myocarditis. Immunity 2017;47:974-89.

9 DeBerge M, Yeap XY, Dehn S, et al. MerTK cleavage on resident cardiac macrophages compromises repair after myocardial ischemia reperfusion injury. Circ Res 2017;121:930-40.

10 Hulsmans M, Clauss S, Xiao L, et al. Macrophages facilitate electrical conduction in the heart. Cell 2017; 169:510-22.

11 Parameswaran N, Patial S. Tumor necrosis factor- $\alpha$ signaling in macrophages. Crit Rev Eukaryot Gene Expr 2010;20:87-103.

12 Van Praet L, Van den Bosch FE, Jacques P, et al. Microscopic gut inflammation in axial spondyloarthritis: a multiparametric predictive model. Ann Rheum Dis 2013;72:414-7.

13 Filimon AM, Negreanu L, Doca M, et al. Cardiovascular involvement in inflammatory bowel disease: Dangerous liaisons. World J Gastroentero/ 2015;21:9688-92.

14 Smolen JS, Breedveld FC, Burmester GR, et al. Treating rheumatoid arthritis to target: 2014 update of the recommendations of an international task force. Ann Rheum Dis 2016:75:3-15.

15 Agca R, Heslinga SC, Rollefstad S, et al. EULAR recommendations for cardiovascular disease risk management in patients with rheumatoid arthritis and other forms of inflammatory joint disorders: 2015/2016 update. Ann Rheum Dis 2017;76:17-28.

16 Chen X, Bäumel M, Männel DN, et al. Interaction of TNF with TNF receptor type 2 promotes expansion and function of mouse CD4+CD25+T regulatory cells. J Immunol 2007;179:154-61.

17 Okubo Y, Mera T, Wang L, et al. Homogeneous expansion of human T-regulatory cells via tumor necrosis factor receptor 2. Sci Rep 2013;3:3153.

18 Ward-Kavanagh LK, Lin WW, Šedý JR, et al. The TNF Receptor Superfamily in Co-stimulating and Coinhibitory Responses. Immunity 2016;44:1005-19.

19 Nguyen DX, Ehrenstein MR. Anti-TNF drives regulatory T cell expansion by paradoxically promoting membrane TNF-TNF-RII binding in rheumatoid arthritis. J Exp Med 2016:213:1241-53.

20 Steeland S, Puimège L, Vandenbroucke RE, et al. Generation and characterization of small single domain antibodies inhibiting human tumor necrosis factor receptor 1. J Biol Chem 2015;290:4022-37. 